\title{
PERCEPTION OF SUSTAINABLE DESIGN INTEGRATION BENEFITS AMONG INDUSTRY PRODUCT DEVELOPMENT TEAMS: A CASE STUDY
}

\author{
T. Chatty ${ }^{1, \otimes}$ and J. Faludi ${ }^{1,2}$ \\ ${ }^{1}$ Dartmouth College, United States of America, ${ }^{2}$ Delft University of Technology, The Netherlands \\ $\triangle$ tejaswini.chatty.th@dartmouth.edu
}

\begin{abstract}
How do employees perceive the impact of incorporating sustainability considerations into their product development practice? In this case study, we observe how these perceptions can be shifted by teaching workshops on how to apply sustainable design methods in practice. We compare the trends for different methods on various dimensions such as creativity, design process time, product marketability etc. Results show an overall shift towards positive perception for all the methods on a majority of factors, indicating a way to ease the adoption of sustainable design into industry practice.
\end{abstract}

Keywords: sustainable design, design methods, industry practice, case study, sustainability

\section{Introduction}

\subsection{Overview}

Sustainable Design focuses on the integration of environmental considerations in product development (Karlsson and Luttropp, 2006). There are many methods and tools that aim to support this integration in unique ways, but as Homans (1949) wrote, "there are neither good or bad methods, only methods that are more or less effective under particular circumstances". Our research investigates how providing employees with the awareness and training to apply the right methods in the right circumstances in practice, can ease the adoption of sustainable design.

Such adoption is critical because of a growing consensus in the industry on the imperative to consider sustainability in the early stages of product development (Chiu and Chu, 2012). Consumers increasingly expect businesses to be sustainable; and in response, big companies like Ford (White, 2019), IKEA (Alänge et al., 2016), Wal-Mart (Meeks and Chen, 2011), and Unilever (Mirvis, 2011) have adopted high-level sustainability initiatives. However, Lamm et al. (2015) argues that paying attention to the role of the individual employees in implementing environmental sustainability is equally important. If employees perceive the impact of incorporating sustainability positively, they may champion and further develop the company's sustainability practice (Ramus and Killmer, 2007).

As part of a case study to test this hypothesis regarding the perception and adoption of initiatives such as those pertaining to sustainability among employees, we conducted workshops on sustainable design methods at an industrial tools and household hardware company that had similarly adopted high-level sustainability initiatives. Employees were instructed on the various methods and taught to apply them 
to problems within their professional contexts. Surveys conducted before and after the workshop quizzed employees on their perception of the impact of incorporating sustainability into their daily practice. Pre-workshop surveys showed that there was a strong negative perception of sustainability's influence on most factors. Perceptions towards sustainability shifted significantly positively on several factors after the employees learned to apply these sustainable design methods to problems relevant to their practice.

\subsection{Design methods evaluated}

This experiment employed four sustainable design methods: Life Cycle Assessment (ISO 14040:2006, 2016), Whole System Mapping (Faludi, 2015), Biomimicry (Baumeister et al., 2013; Benyus, 1997), and The Natural Step (Robèrt, 1991; Baxter et al., 2009). These methods were chosen for their diversity in approach to sustainable design, and based on recommendations gathered in a past study from professional designers, engineers, and managers working in product development across industry sectors (Faludi et al., 2019; Faludi and Agogino, 2018). The workshop contents were put together as part of the author's dissertation (Faludi, 2017). The following is a brief overview of the methods, excerpted from the workshop content, in the order that they were conducted:

\subsubsection{Life Cycle Assessment}

Life cycle assessment, as defined by the ISO standards, is "the compilation and evaluation of the inputs, outputs, and the potential environmental impacts of a product system throughout its life cycle" (ISO 14040:2006, 2016). The method involves four stages: goal and scope definition, inventory analysis, impact assessment, and interpretation of results, which are undertaken in an iterative process, to quantitatively compute and visualize the environmental impacts of different product ideas.

\subsubsection{Whole System Mapping}

Whole System Mapping is a sustainable design and innovation method which begins with the participants collaboratively drawing a visual map of the problem space. Life cycle assessment results and business constraints are employed to set priorities for requirements. Finally, participants use the whole system map, the prioritized requirements, and estimated LCA results to select one or more design ideas (Faludi, 2015), (Faludi, 2018).

\subsubsection{Biomimicry}

Biomimicry can be applied in a variety of ways, and the version that was taught in the workshops followed the methodology prescribed by Faludi and Menter (2013). The design problem is first redefined biologically, after which participants attempt to identify relevant biological strategies for inspiration using both real artefacts and on the AskNature.org online repository (Deldin and Schuknecht, 2014). These strategies are translated into tangible product features, and tested against the Biomimicry Institute's list of "Nature's Principles" (Baumeister et al., 2013) for compatibility with nature.

\subsubsection{The Natural Step}

The Natural Step uses "sustainability principles" (Baxter et al., 2009) to define a perfect sustainability vision and build awareness, and then use backcasting to approach it. A gap analysis is conducted to identify critical sustainability issues where reality is far removed from the vision. Participants then brainstorm to generate creative solutions to bridging the gaps. The final step uses the criteria of return on investment, platform versus one-time product, and alignment with the vision to decide which new ideas to move forward with.

\section{Methodology}

In this section, we describe our study's procedure and participants, followed by details about administered surveys, and finally the metrics used to assess the outcomes. 


\subsection{Participants and procedures}

For this study, we worked with a large company in the industrial tools and household hardware domain whose top management had recently initiated several high-level sustainability policies. We sought to test our hypothesis that employee perception of the impact of making this change would be negative, which could prove to be a critical barrier to adoption. We further wanted to evaluate the shift in perception upon educating employees to apply the right methods and tools within the context of their practice. To this effect, four workshops were conducted at the company's office on the following sustainable design methods: Life Cycle Assessment, Whole System Mapping, Biomimicry, and The Natural Step. Each workshop lasted two hours and constituted two sections: instruction, and application. The 45-60 minute instruction section involved a PowerPoint presentation and discussion led by the principal investigator to provide participants with an overview of the method. This was followed by a 60 -minute application section, where participants worked in teams to apply the method to a self-defined problem statement relevant to their practice. Participants were asked to pick a specific problem that one of them had encountered as part of a recent project, allowing them to recognize the difference in perspective when applying a sustainable design method. The graduate student who is the first author of this paper helped oversee how the teams were applying the method and assisted with queries.

The workshops were advertised by the sustainability office across the company, and the participants constituted employees who had volunteered to participate. The workshops attracted a good mix of individuals who worked in a variety of areas such as: design, various engineering fields, packaging, supply-chain management, sales, and general management.

There were a total of 80 participants overall. Participants were allowed to sign up for multiple workshops which allowed for an overlap in the audience for each workshop. They were requested to fill out a pre-workshop survey and a post-workshop survey in order to evaluate their perception of how integrating sustainable design into their practice would affect a list of factors. They also rated which specific components within each method they found to be valuable.

A total of 33 participants responded to the pre-workshop survey and 35 responded to the postworkshop surveys. For the post-workshop survey, the number of responses per method include: 11 participants for life cycle assessment, 8 participants for biomimicry, 10 participants for whole system mapping, and 6 participants for The Natural Step. All surveys were anonymous, and no records of participant attendance were kept in compliance with the confidentiality agreement with the company. This prevents us from identifying the extent of overlap of participants across workshops, or trace perception change before and after the workshop individually by participant.

\subsection{Survey design}

One of the goals of this case study was to evaluate the participants' perception of how incorporating sustainability into their practice would impact their workflow along the following factors: design process time, design process cost, final product cost, creativity of the product/solution, product quality, product marketability, ease of manufacturing, and overall business value. Participants were surveyed before and after the workshops to gauge how the workshops influenced their perception along these dimensions.

We hypothesized that providing employees with a toolbox of methods and teaching them how to apply the tools in the right context can shift their perception positively, thereby improving adoption. Since each design method has different strengths and weaknesses, we expected to see differences in trends across methods.

\subsubsection{Pre-workshop survey}

The pre-workshop survey asked participants how systematically and how often their teams considered sustainability when making design, manufacturing, or strategic decisions. They were also surveyed about their role in the company, and the size of their team, to help us gain a better understanding of their organizational dynamics.

Participants were then asked to rate how they believed incorporating sustainability would affect the set of factors listed above. They rated their perceptions for each of these factors on a Likert Scale ranging 
from: much worse, slightly worse, no change, slightly better, much better. The entire pre-survey comprised of 7 questions and took approximately 15 minutes to complete.

\subsubsection{Post-workshop survey}

The post-workshop survey started with a variation on the pre-survey questions about how participants perceived sustainability would affect the earlier set of factors, except, rather than asking how sustainability generally affected the aforementioned list of factors, the post survey asked how implementing the workshop's specific design method would affect those factors.

We compared the responses of the pre- and post-workshop surveys to evaluate if there was an overall shift in perception before and after the workshop, and if there were differences in this shift across participants who attended different workshops. The results showed a significant shift in overall perception and interesting trends across methods, validating our hypotheses.

The post-workshop survey also asked participants about how individual components/steps in each method provided them with different kinds of value. For example, Life cycle assessment constitutes the following components/steps: goal and scope definition, choosing functional units, determining inventory, impact assessment, and the interpretation of results. Participants were asked which of these components offered them which of the following kinds of value: overall value, sustainability value, innovation value, or business value. This adds data to past studies on how components/steps within sustainable design methods can be treated as separate tools in a toolbox, which can further be tailored to the company's workflow.

\subsection{Defining the evaluation criteria}

This section defines the list of evaluation criteria as explained to the participants, utilized to evaluate their perceptions of the impact of incorporating sustainability. This list of criteria was compiled through discussions with industry experts on what aspects are critical to the adoption of sustainable design methods into regular practice (Faludi and Agogino, 2018).

1. Design Process Time (DPT): compares how much time the participant's team might spend on the project in product development.

2. Design Process Cost (DPC): combines the estimated cost of additional time, expert labour, specialized software, or other costs in product development.

3. Final Product Cost (FPC): compares an increase or decrease in the final cost of the product to customers.

4. Creativity (CRE): is defined by evaluating whether the ideas/solutions generated are novel, purposeful, and resource effective (Chakrabarti, 2006).

5. Product Quality (PQ): compares an increase or decrease in quality of final product, as measured by traditional dimensions such as performance, features, reliability, conformance, durability, serviceability, aesthetics, and perceived quality (Garvin, 1984).

6. Product Marketability (PM): is measured by an increase or decrease in desirability and brand value to customers.

7. Ease of Manufacturing (EoM): is defined by an increase or decrease in manufacturing cost and/or manufacturing complexity.

8. Overall Business Value (OBV): combines all of the above criteria for an overall estimate of increased or decreased profitability. Judgement of this factor can vary across participants and teams.

\section{Preliminary Results}

\subsection{Graphs}

\subsubsection{Comparison of overall perception}

Figure 1 compares the shift in the overall perception. For this initial study with limited data, a 5\% error bar was chosen as a baseline to derive insights. This is done with the recognition that it could lead to false correlations, and hence, all insights are validated through discussions among the research 
team and interviews with volunteering participants. This graph does not capture the differences in shift across the four design methods, which will be discussed in the next section.

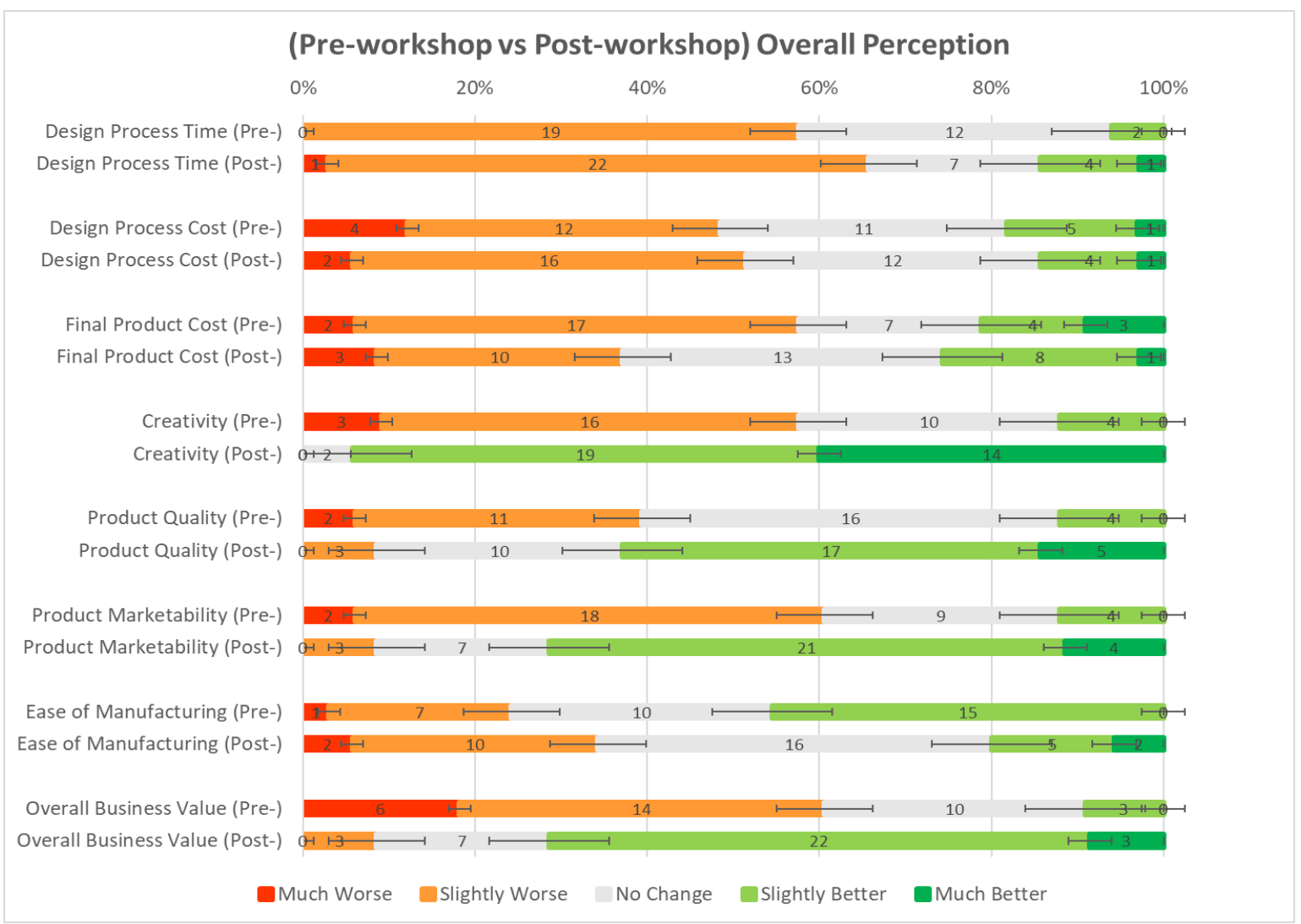

Figure 1. Shift in overall perception of the impact of incorporating sustainability

Specifically, we observe the following shifts across each of the factors pre- and post- workshops:

- Design Process Time (DPT): The shift in percentages of positive and negative impact on design process time are not statistically significant.

- Design Process Cost (DPC): We observe a statistically significant decrease in percentage of responses claiming 'much worse' (negative) impact. The overall shift in positive vs negative impact after the workshop is not significant.

- Final Product Cost (FPC): The graph shows a statistically significant decrease in the overall negative impact after the workshop.

- Creativity (CRE): This factor shows the greatest shift in perception of impact. Perceptions of negative impact before the workshops shifted almost entirely to perceptions of positive impact after the workshop.

- Product Quality (PQ), Product Marketability (PM), and Other Business Value (OBV): These factors display a similar trend: a statistically significant increase in perceived positive impact, coupled with a significant decrease perceived negative impact.

- Ease of Manufacturing (EoM): The graph depicts a significant decrease, by more than $20 \%$, in perception of positive impact resulting in a significant increase in percentage of participants claiming 'no change'.

\subsubsection{Comparing trends across design methods}

Graphs in Figure 2 and Figure 3 compare the shift in perception for each factor and each design method, before and after the workshops. These results help identify the roots of the overall shifts observed in the previous section, while depicting which design method shifts perception of impact on which corresponding factors. 


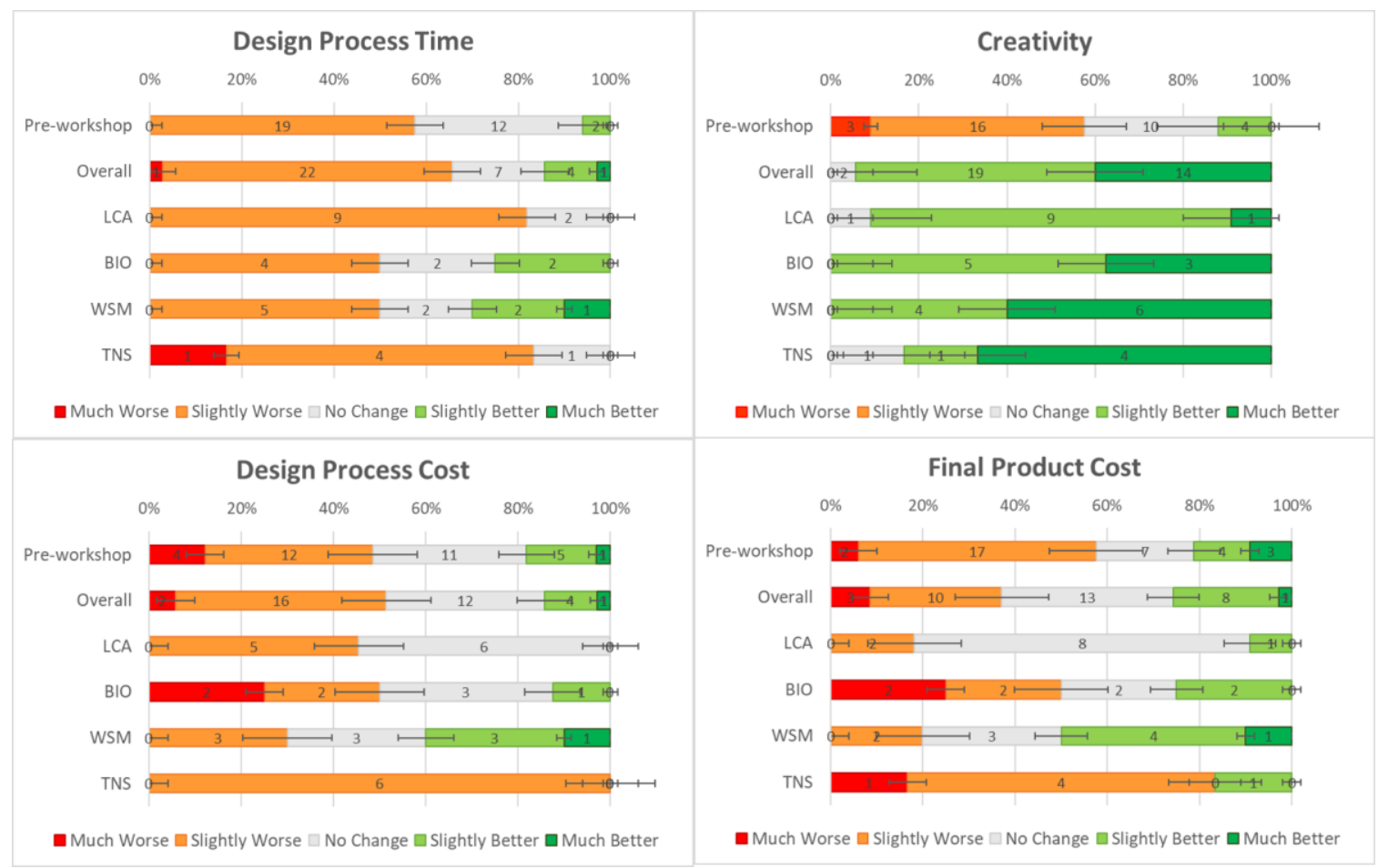

Figure 2. Shift in perceptions across design methods (1)

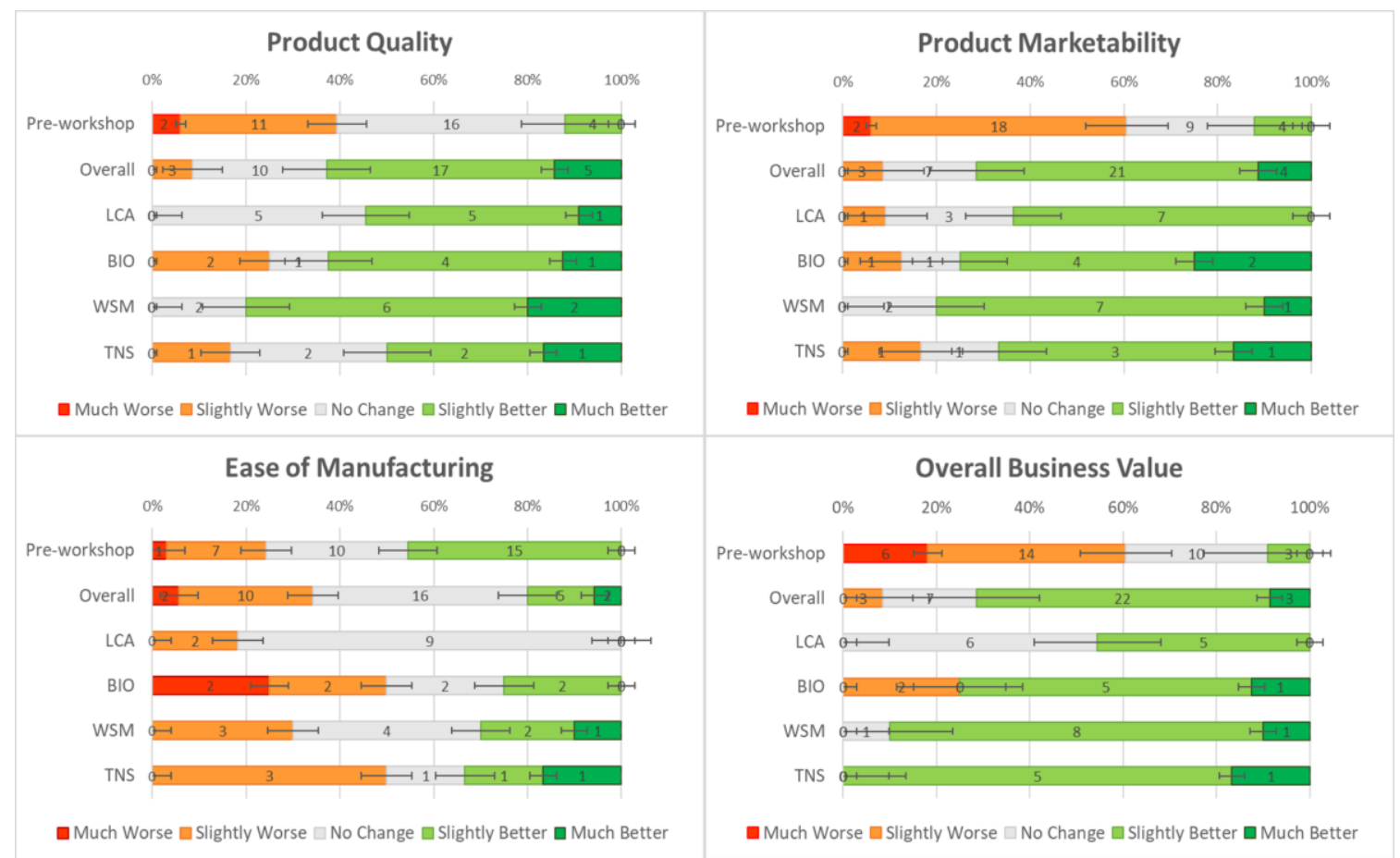

Figure 3. Shift in perceptions across design methods (2)

Observations in trends across the above factors before and after the workshops, categorized by specific design method workshop:

- Design Process Time:

- There is a statistically significant increase in perceived negative impact by nearly $20 \%$ among participants who attended the LCA and The Natural Step workshops. 
- There is also a significant increase in perceived positive impact for the Whole System Mapping and Biomimicry workshops, but only by a margin of approximately $10 \%$.

- Design Process Cost:

- There is a very large increase in perceived negative impact for The Natural Step, and approximately twice the perceived positive impact for Whole System Mapping.

- Final Product Cost:

- Similar to Design Process Cost, there is a statistically significant increase in perceived negative impact for the Natural Step workshop

- For Whole System Mapping, positive perceptions were more than doubled and negative perceptions fell by almost $2 / 3$ rds; given the importance of final product cost, this may be a strong driver for company-wide adoption of sustainability in design.

- A significant percentage of participants who attended the LCA workshop thought it would not impact Final Product Cost.

- We also observe an increase in percentage of participants who marked 'much worse' and a corresponding decrease in percentage of participants who marked 'much better' in the case of Biomimicry, without a difference in overall positive vs negative impact margins.

- Creativity:

- There seems to be a significant increase in perceived positive impact on Creativity among all participants regardless of the design method. There is no perceived negative impact across all of the design methods. This graph depicts one of the most significant trends in the data from this experiment, and is highly encouraging for the adoption of sustainability in product development.

- Product Quality and Product Marketability:

- There is a statistically significant increase in perceived positive impact and decrease in perceived negative impact on product quality and product marketability across all the design methods. The shift is not as sweeping as in the case of creativity, but is still encouraging.

- Ease of Manufacturing:

- There appears to be a significant increase in perceived negative impact in the case of both biomimicry and The Natural Step. This is accompanied by a decrease in perceived positive impact across all the design methods, compared to the pre-workshop survey.

- LCA is overwhelmingly perceived as not impacting ease of manufacturing, which is accurate, as it does not prescribe any particular manufacturing methods or materials, but simply quantifies the environmental impact of existing designs.

- Overall Business Value:

- The trends display a significant decrease in perceived negative impact and a significant increase in perceived positive impact, across all design methods.

- The greatest increase in perceived positive impact comes from the natural step, where the percentage of participants who perceived negative impact is zero.

\subsection{Key observations and implications}

Figure 1 revealed some key insights in overall perceptions:

- Creativity: Before the workshops, over half of the respondents perceived sustainability as hurting product creativity, but after the workshops, the overwhelming majority reported that the sustainable design methods they learned were in fact beneficial to creativity. This suggests that the simple exposure to how to apply the right tools can reverse professionals' perceptions of the correlation between sustainability and creativity and in turn encourages adoption.

- Product Quality, Marketability, and Overall Business Value: These factors all showed an increase in perceived positive impact coupled with a decrease in perceived negative impact. This trend is reflected in the shifts across all design methods, pointing to how prejudices about sustainability may be dispelled by empowering employees with greater awareness. 
Figure 2 and 3 revealed more specific insights about the different sustainable design methods:

- The Natural Step showed the greatest increase in positivity for Overall Business Value as its perceived negative value was reduced to zero. This may mean it is more attractive to formulating business strategy than the other methods tested.

- Whole System Mapping improved perceptions of design process cost and final product cost far more than the other methods; it also performed better on product quality, although the differences were not always statistically significant. This may mean its adoption is more likely to be profitable than the other methods tested, and can be expected to be adopted by those in product management roles.

Altogether, our results led to the formulation of the following hypotheses that go beyond the evaluation of perception. We seek to test these in our future research:

- Sustainable design methods are not merely subjectively perceived to improve creativity in product development, but they actually can objectively improve it. Design ideas from a product development cycle in which sustainable design methods are employed will, on average, be more creative than those from a regular product development cycle, when judged by impartial reviewers.

- Whole system mapping improves product quality, design process cost, and final product cost, while The Natural Step improves overall business value, not just in perception but also in reality.

- Biomimicry improves marketability, not just in perception, but in reality.

\section{Limitations and Scope for Future Work}

The study was conducted with a total of 80 participants, but only 33 participants responded to the preworkshop survey and 35 participants responded to the post-workshop survey. This was due to the fact that participants were encouraged to respond to surveys but it was not made mandatory, and several had to rush to their next meeting immediately after the workshops. These small sample sizes cause us to consider these outcomes to be preliminary results that help to formulate hypotheses guiding future investigation. In addition, there may be a selection bias, where those who felt less positively about the workshops did not fill out the surveys. We seek to conduct similar experiments at multiple companies, with higher percentages of survey respondents including those with sceptical initial perceptions, in order to gather more data to test the hypotheses suggested in this paper. We hope to conduct much more extensive statistical analyses on the expanded dataset.

This case study was conducted at a single company which produces industrial tools and household hardware. Trends identified from this experiment may not necessarily hold true in the context of a different industry sector. We are currently working with companies in other industry sectors to expand the scope and validate our findings. We highly encourage other researchers to test these and other sustainable design methods with a broad range of companies.

\section{Conclusion}

This experiment explored the perception among professionals regarding the impact of incorporating sustainability into their product development practice. We sought to observe if teaching specific sustainable design methods, and helping professionals apply the method to a case relevant to their practice would cause a shift in this perception. Results showed that there was indeed a shift towards positive perception for a majority of the parameters, overall, and across each design method. All of the methods taught improved perceptions of product/solution creativity. Whole System Mapping was also found to improve perceptions of product quality, product marketability, design process cost, and final product cost. The Natural Step was perceived to improve overall business value, while Biomimicry was perceived to improve marketability.

While this paper compares the differences in trends across each design method, it does not aim to suggest that one method is better or worse than the other. It instead promotes using these distinctions as a guide to selecting the right tool for the job. The paper also highlights how when a top-down approach to incorporating sustainability is mandated, it could lead to negative perceptions of how it would impact the workflow. When employees are instead made aware and trained on applying the 
right tools in the right context, this can lead to a more positive attitude to the integration of sustainability into product development practice.

\section{References}

Alänge, S., Clancy, G. and Marmgren, M. (2016), "Naturalizing sustainability in product development: A comparative analysis of IKEA and SCA", Journal of Cleaner Production, Vol. 135, pp. 1009-1022. https://doi.org/10.1016/j.jclepro.2016.06.148

Baumeister, D. et al. (2013), Biomimicry Resource Handbook: A Seed Bank of Best Practices, 2013 edition, Biomimicry 3.8, Missoula, MT.

Baxter, K. et al. (2009), Sustainability Primer: Step By Natural Step, The Natural Step Canada.

Benyus, J.M. (1997), Biomimicry, William Morrow, New York.

Chakrabarti, A. (2006), "Defining and Supporting Design Creativity", 9th International Design Conference, DESIGN, pp. 479-486.

Chiu, M.-C. and Chu, C.-H. (2012), "Review of sustainable product design from life cycle perspectives", Int. J. Precis. Eng. Manuf., Vol. 13, pp. 1259-1272. https://doi.org/10.1007/s12541-012-0169-1

Deldin, J.-M. and Schuknecht, M. (2014), "The AskNature Database: Enabling Solutions in Biomimetic Design", In: Goel, A.K., McAdams, D.A. and Stone, R.B. (Eds.), Biologically Inspired Design: Computational Methods and Tools, Springer, London, pp. 17-27. https://doi.org/10.1007/978-1-4471-5248-4_2

Faludi, J. (2018), Whole System Mapping - Exercise. VentureWell Tools for Design and Sustainability. Available at: https://venturewell.org/tools_for_design/whole-systems-mapping/whole-systems-mappingexercise/ (accessed 10.11.18).

Faludi, J. (2017), Golden Tools in Green Design: What drives sustainability, innovation, and value in green design methods? [PhD Thesis], UC Berkeley.

Faludi, J. (2015), "A Sustainable Design Method Acting as an Innovation Tool”, In: ICoRD'15-Research into Design Across Boundaries Volume 2, Springer, pp. 201-212.

Faludi, J. and Agogino, A.M. (2018), "What Design Practices Do Professionals Use For Sustainability And Innovation?" DS92: Proceedings of the DESIGN 2018 15th International Design Conference, Dubrovnik, Croatia, pp. 2633-2644.

Faludi, J. et al. (2019), "Preliminary Results Testing What Different Design Solutions Arise from Different Sustainable Design Methods", Proceedings of the Design Society: International Conference on Engineering Design, Vol. 1, pp. 3351-3360. https://doi.org/10.1017/dsi.2019.342

Faludi, J. and Menter, A. (2013), "Sustainable Design Education Through a Self-Directed Online System", ASME 2013 International Design Engineering Technical Conferences and Computers and Information in Engineering Conference, American Society of Mechanical Engineers, pp. V004T05A033-V004T05A033.

Garvin, D.A. (1984), "Product quality: An important strategic weapon", Business Horizons, Vol. 27, pp. 40-43. https://doi.org/10.1016/0007-6813(84)90024-7

Homans, G.C. (1949), “The strategy of industrial sociology", American Journal of Sociology, Vol. 54, pp. 330-337.

ISO 14040:2006 (2016), Environmental management - Life cycle assessment - Principles and framework, 2nd ed, ISO.

Karlsson, R. and Luttropp, C. (2006), "EcoDesign: what's happening? An overview of the subject area of EcoDesign and of the papers in this special issue", Journal of Cleaner Production, Vol. 14, pp. 1291-1298. https://doi.org/10.1016/j.jclepro.2005.11.010

Lamm, E., Tosti-Kharas, J. and King, C.E. (2015), "Empowering Employee Sustainability: Perceived Organizational Support Toward the Environment", J Bus Ethics, Vol. 128, pp. 207-220. https://doi.org/1 0.1007/s10551-014-2093-z

Meeks, M. and Chen, R. (2011), Can Walmart Integrate Values with Value?: From Sustainability to Sustainable Business. Retail, Hospitality, and Tourism Management Publications and Other Works.

Mirvis, P. (2011), "Unilever's Drive for Sustainability and CSR - Changing the Game", In: Organizing for Sustainability, Emerald Group Publishing Limited, pp. 41-72.

Ramus, C.A. and Killmer, A.B.C. (2007), "Corporate greening through prosocial extrarole behaviours - a conceptual framework for employee motivation", Business Strategy and the Environment, Vol. 16, pp. 554570. https://doi.org/10.1002/bse.504

Robèrt, K.-H. (1991), “Educating A Nation: The Natural Step", Context, Vol. 28, p. 10.

White, R. (2019), Ford Motor Company Reflects on 20 Years in Sustainability with New Goals Ahead [WWW Document]. Available at: https://www.wkar.org/post/ford-motor-company-reflects-20-years-sustainabilitynew-goals-ahead (accessed 10.26.19). 
